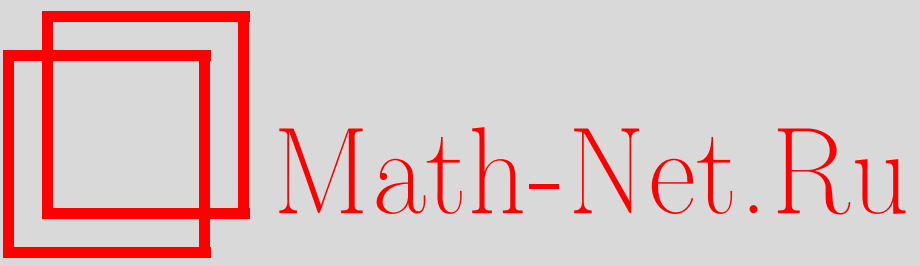

В. В. Лидский, Уравнение движения излучающей заряженной частицы в классической электродинамике, ТМФ, 2005, том 143, номер 1, 112-130

DOI: https://doi.org/10.4213/tmf1806

Использование Общероссийского математического портала Math-Net.Ru подразумевает, что вы прочитали и согласны с пользовательским соглашением

http://www . mathnet.ru/rus/agreement

Параметры загрузки:

IP : 44.207 .124 .84

26 апреля 2023 г., 14:44:57 
ТЕОРЕТИЧЕСКАЯ

И МАТЕМАТИЧЕСКАЯ

ФИЗИКА

Том 143, № 1

апрель, 2005

(C) 2005 г.

В. В. Лидский*

\title{
УРАВНЕНИЕ ДВИЖЕНИЯ ИЗЛУЧАЮШЕЙ ЗАРЯЖЕННОЙ ЧАСТИЦЫ В КЛАССИЧЕСКОЙ ЭЛЕКТРОДИНАМИКЕ
}

\begin{abstract}
Предложены классические уравнения движения заряженных частиц в электромагнитном поле, содержащие общие формулы для ускорения частицы, учитывающие торможение излучением и не содержащие вторых производных скорости частицы. В pяде рассмотренных частных случаев новые уравнения приводят к результатам, совпадающим с известными в литературе и проверенными на опыте. Показано, что в диапазоне сверхвысоких энергий классическая электродинамика не приводит к внутренним противоречиям и допускает в принципе движение частиц с энергиями, превосходящими предел Померанчука.
\end{abstract}

Ключевые слова: классическая электродинамика, электромагнитная масса, радиационное трение, реакция излучения, торможение излучением, уравнение Лоренца-Дирака.

\section{1. ВВЕДЕНИЕ}

Вопрос об уравнении движения заряженной частицы при учете силы радиационного трения Гинзбург отнес к "вечным вопросам", обсуждаюшимся в науке десятилетиями [1]. К этой же категории относится и проблема электромагнитной массы, тесно связанная с проблемой радиационного трения. Кроме названной монографиии [1], содержашей богатую библиографию, обзоры этих проблем можно найти в работах [2], [3] и в недавно вышедшей статье [4].

Уравнение движения электрона, учитываюшее радиационное трение, было предложено Лоренцем в рамках теории эфира [5]. Абрагам получил выражения для электромагнитной массы, энергии и импульса поля электрона [6]. В 1923г. Ферми предложил способ вычисления электромагнитной массы, энергии и импульса в геометрии Минковского и показал, что в случае сферически-симметричного распределения зарядов электромагнитные энергия и масса удовлетворяют релятивистскому соотношению $\mathcal{E}=m c^{2}$, а релятивистские энергия и импульс поля, связанного с движущимся зарядом, образуют относительно преобразований Лоренца 4-вектор $P^{i}$, причем $P^{i} P_{i}=m^{2} c^{4}[7]$. Дирак

* Физический институт им. П. Н. Лебедева РАН, Москва, Россия. E-mail: vlidsky@sci.lebedev.ru 
предложил релятивистское уравнение движения классического электрона [8], получившее название уравнения Лоренца-Дирака (ЛД-уравнение):

$$
m a^{i}=e F^{i l} u_{l}+\frac{2}{3} e^{2} \dot{a}^{i}+\frac{2}{3} e^{2} a^{k} a_{k} u^{i},
$$

где мы использовали обозначения, принятые в книге [9]: латинские индексы пробегают значения $0,1,2,3 ; u^{i}$ и $a^{i}$ - соответственно 4-скорость и 4-ускорение частицы; точка над буквой обозначает дифференцирование по инвариантному собственному времени частицы $\tau$ :

$$
a^{i} \equiv \dot{u}^{i} \equiv \frac{d u^{i}}{d \tau}, \quad \dot{a}^{i} \equiv \frac{d a^{i}}{d \tau}
$$

$e$ и $m$ - заряд и масса частицы, соответственно; $F^{i k}$ - тензор внешнего электромагнитного поля. Скорость света мы приняли равной единице. Далее мы будем пользоваться этими обозначениями.

В последние несколько лет появился ряд работ, в центре внимания которых стоит уравнение Л Д, причем наряду с работами, анализирующими его в традиционном ключе (см. [10]), некоторые авторы ищут новые подходы [11].

Авторы работ [12]-[14] критически относятся к ЛД-уравнению и предлагают его модификации. Уравнение движения безмассовой частицы предложено в работе [15].

К существенным недостаткам ЛД-уравнения Рорлих [13] относит, во-первых, то, что уравнение (1) третьего порядка, в связи с чем задание начальных координат и скоростей частиц не определяет однозначно движения. Второй, более существенньй недостаток наличие решений с экспоненциально возрастаюшей скоростью при отсутствии внешней силы (так называемые самоускоряюшиеся решения [8]). Третий - наличие излучения и индуцированного самодействия при равенстве нулю внешней силы.

Мы предложим в данной работе уравнение движения заряженной частицы, учитьвающее реакцию излучения и свободное от трех перечисленных недостатков.

Как подчеркнуто в монографии [1], проблема реакции излучения связана с проблемой энергии в электродинамике. То обстоятельство, что уравнение (1) в принципе допускает самоускоряюшиеся решения, свидетельствует о том, что в нем сокрыто противоречие с законом сохранения энергии. При этом часто упоминаемоев литературе дополнительное требование малости силы радиационного трения по сравнению с внешней силой не снимает этого возражения, ибо мы хотим построить теорию, в которой закон сохранения энергии был бы абсолютно точным законом природы, не допускающим даже самого малого нарушения.

Начнем с того, что дадим рабочую формулировку закона сохранения энергии, свободную от расходящихся несобственных интегралов. Мы используем затем эту формулировку для получения уравнения движения частиц, вычислив поток тензора энергии-импульса через поверхность, окружающую траекторию частищы. По существу, мы повторим программу расчета, выполненного в работе [8]. Главное отличие нашего расчета заключается в следующем: мы примем, что поле вблизи траектории частицы определяется не разностью запаздывающего и опережающего потенциалов, а исключительно запаздьвающими потенциалами Лиенара-Вихерта. Кроме того, поток 
энергии-импульса мы вычислим через специальным образом сконструированную поверхность трубки вокруг мировой линии частицы, благодаря чему удастся вьгислить точные значения всех интегралов; мы не будем пользоваться разложением поля в ряд Тейлора, приводящим к появлению в ответе второй производной скорости частищ [8].

Затем мы сравним полученный результат с частными случаями излучения частиц при движении в электромагнитных полях, известными по литературе и проверенными экспериментально.

Особо рассмотрим движение частищ сверхвысоких энергий с точки зрения классической теории. Хотя в этой области энергий классическая электродинамика не применима из-за квантовых эффектов, принципиально важно, что последовательная классическая теория и здесь не сталкивается с внутренними противоречиями и не приводит к решениям, содержащим нарушение закона сохранения энергии.

Частицы мы будем рассматривать как точечные. Наиболее удобным математическим объектом, соответствующим точечной классической частице, мы считаем особые точки функций координат, описывающих электромагнитное поле. В общей теории относительности представление о частицах как об особых точках пространства-времени рассматривалось Эйнштейном и Инфельдом [16] (см. также имеющиеся там ссылки на более ранние работы). В рамках такого подхода логично считать, что особые точки напряженности поля являются одновременно особыми точками пространства-времени. В этом случае нам не придется вводить в теорию дополнительные параметры частищы, помимо заряда и массы, что неизбежно в моделях частиц конечных размеров. Мы обойдем известные парадоксы, если не станем ставить вопросы о значении поля "в точке, где находится частища" или о "полной энергии частицы”, что вполне естественно в модели Эйнштейна-Инфельда.

\section{2. О ЗАКОНЕ СОХРАНЕНИЯ ЭНЕРГИИ}

Примем следуюшую формулировку закона сохранения әнергии.

Всякой гладкой замкнутой пространственноподобной 2-поверхности $S$, ограничивающей 3-объем $V$ Вселенной, может быть поставлен в соответствие 4-вектор $P^{i}$, являющийся вектором энергии-импульса 3-объема $V$, причем $V$ может либо содержать частищы, либо не содержать частиц. Энергия не может ни возникать, ни исчезать, а только передаваться от одного объема к другому через обшую границу.

Отсюда следует, что может быть определен 4-тензор плотности потока энергии-импульса $T^{i k}$, так что изменение со временем энергии 3-объема $V$, принадлежащего 3 -пространству $t=$ const, связано с потоком тензора $T^{i k}$ через 2 -поверхность $S$, ограничиваюшую $V$ :

$$
\frac{d}{d t} P^{i}=-\oint_{S}\left(\vec{T}^{i}, \vec{n}\right) d S
$$

где 3-векторы $\vec{T}^{i}$ образованы пространственными компонентами 4-тензора $T^{i k}$ и представляют собой поток $i$-й компоненты энергии-импульса $P^{i} ; \vec{n}-3$-вектор внешней нормали к поверхности $S$. 
В соответствии с упомянутым выше взглядом на частицы как особые точки пространства-времени мы считаем, что на гладкой поверхности $S$ частиц нет, в связи с чем интеграл (2) всегда интеграл собственный.

Относительно энергии-импульса частиц мы примем следуюшее естественное предположение: при непрерывной деформации поверхности $S$ соответствуюший вектор энергии-импульса $P^{i}$ изменяется непрерывно. При попадании частицы внутрь $S$ величина $P^{i}$ изменяется скачкообразно, причем величина этого скачка $\Delta P^{i}$ может быть неограниченно большой; скачок $\Delta P^{i}$ зависит от типа частицы и не может быть определен в обшей теории электродинамики.

Определение (2) мы выписали в системе отсчета наблюдателя, неподвижного относительно поверхности $S$. Несложно придать ему релятивистски-инвариантную форму. Рассмотрим пространственноподобную гладкую замкнутую 2-поверхность $S$. Будем непрерывно деформировать $S$, не пересекая при деформации мировых линий частиц. Обозначим через $\bar{S}$ деформированную поверхность $S$, а через $\Sigma$ - 3 -гиперповерхность, заметенную $S$ при деформации. Согласно закону сохранения энергии-импульса можно утверждать, что разность величин $P^{i}$ на поверхностях $\bar{S}$ и $S$ равна потоку тензора плотности потока энергии-импульса $T^{i k}$ через 3 -поверхность $\Sigma$ :

$$
P^{i}(\bar{S})-P^{i}(S)=-\int_{\Sigma} T^{i k} d V_{k}
$$

где $d V_{k}=e_{k l m n} d x^{l} d x^{m} d x^{n}$ - элемент интегрирования, направленный вдоль 4-нормали к 3 -поверхности $\Sigma$. Направление 4 -нормали $n_{k}$ к $\Sigma$ мы выбираем так, чтобы на 2-границах $\Sigma$ пространственные компоненты нормали совпадали с компонентами внешних нормалей к 2-поверхностям $S$ и $\bar{S}$.

Разность, стоящая в левой части уравнения (3), не должна зависеть от способа деформирования $S$ в $\bar{S}$. Рассмотрим две 3 -поверхности $\Sigma$ и $\Sigma^{\prime}$, заметаемые 2-поверхностью $S$ при двух различных ее деформациях, приводяших к $\bar{S}$. Разность между потоками тензора $T^{i k}$ через $\Sigma$ и $\Sigma^{\prime}$ равна нулю:

$$
\int_{\Sigma} T^{i k} d V_{k}-\int_{\Sigma^{\prime}} T^{i k} d V_{k}=0
$$

Данная разность по теореме Гаусса может быть преобразована к интегралу от дивергенции $T^{i k}$ по 4-объему $\Omega$, заключенному между 3 -поверхностями $\Sigma$ и $\Sigma^{\prime}$ :

$$
\int_{\Omega} \partial_{k} T^{i k} d \Omega=0
$$

Поскольку $\Omega$ - произвольный объем, свободньй от частиц, мы приходим к выводу о равенстве нулю 4-дивергенции тензора $T^{i k}$ :

$$
\partial_{k} T^{i k}=0 .
$$


Для тензора потока энергии-импульса электромагнитного поля мы воспользуемся выражением Максвелла-Хевисайда (см. [9], § 33):

$$
T^{i k}=\frac{1}{4 \pi}\left(-F^{i l} F_{l}^{k}+\frac{1}{4} g^{i k} F_{l m} F^{l m}\right) .
$$

Здесь $F^{i k}$ - 4-тензор электромагнитного поля (см. [9], $\left.\S 23\right), g^{i k}$ - метрический тензор. Легко убедиться, что в силу уравнений Максвелла с нулевой правой частью для тензора (5) справедливо уравнение (4).

\section{3. ПОЛЕ В ОКРЕСТНОСТИ КУЛОНОВСКОЙ ЧАСТИЦЫ}

Рассмотрим заряженную частицу, движушуюся во внешнем электромагнитном поле. Уравнение движения частицы получим, вычислив интеграл (3) по подобранной подходящим образом 3 -поверхности $\Sigma$, окружающей мировую линию частицы.

В окрестности мировой линии частицы поле может быть представлено в виде суммы $F^{i k}+G^{i k}$, где $G^{i k}$ имеет особенность на мировой линии и исчезает на бесконечности: $G^{i k} \rightarrow 0$ при $R \rightarrow \infty$. Поле $F^{i k}$ регулярно в окрестности частишы и в обшем случае не исчезает при удалении от заряда. Поле $F^{i k}$ естественно интерпретировать как внешнее поле, в котором движется частица, тогда $G^{i k}$ - поле, связанное с само́й заряженной частицей.

В данной работе мы сочли целесообразным не рассматривать многообразие частиц, а ограничиться изучением движения во внешних полях гипотетических кулоновских частиц, все свойства которых определяются двумя параметрами: зарядом и массой. В системе сопутствуюшего наблюдателя поле такой частищы имеет вид

$$
\vec{E}=e \frac{\vec{R}}{R^{3}}, \quad \vec{H}=0 .
$$

Кулоновский член медленнее других убывает на больших расстояниях, в связи с чем именно он в первую очередь определяет взаимодействие. Принимая простейшую модель частицы, мы исходим из предположения, что учет моментов высоких порядков приведет к малым поправкам к уравнениям движения, которые будут получены ниже [3].

Поле $G^{i k}$ в окрестности движущейся кулоновской частицы определяется запаздывающими потенциалами Лиенара-Вихерта, которое удобно записать в четырехмерной форме:

$$
G^{i k}=\frac{e}{\left(\chi^{j} u_{j}\right)^{3}}\left(\chi^{i} u^{k}-u^{i} \chi^{k}\right)\left(1-\chi^{m} a_{m}\right)+\frac{e}{\left(\chi^{j} u_{j}\right)^{2}}\left(\chi^{i} a^{k}-a^{i} \chi^{k}\right) .
$$

Здесь $\chi^{i}=x^{i}-z^{i}-4$-вектор из “точки излучения" $z^{i}$ в "точку наблюдения" $x^{i} ; u^{i}$ и $a^{i}$ - соответственно 4-скорость и 4-ускорение в "точке излучения". Опережаюшие потенциалы мы положим равными нулю.

Если подставить в выражение (5) сумму $F^{i k}+G^{i k}$ вместо $F^{i k}$, то выражение для тензора энергии-импульса распадается на три слагаемых:

$$
T^{i k}=T_{F F}^{i k}+T_{F G}^{i k}+T_{G G}^{i k} .
$$


Здесь $T_{G G}^{i k}$ - члены тензора энергии-импульса, квадратичные по полю $G^{i k}$ и описываюшие движение заряда в отсутствие поля, $T_{F F}^{i k}$ - члены, квадратичные по $F^{i k}$ и соответствуюшие тензору энергии-импульса внешнего поля; $T_{F G}^{i k}$ включает члены, линейные по $F^{i k}$ и $G^{i k}$, и описывает взаимодействие заряда с внешним полем:

$$
T_{F G}^{i k}=\frac{1}{4 \pi}\left(-G^{i} F^{k l}-F^{i l} G^{k}{ }_{l}+\frac{1}{2} g^{i k} G_{l m} F^{l m}\right) .
$$

Формулы для $T_{F F}^{i k}$ и $T_{G G}^{i k}$ по внешнему виду совпадают с выражением (5).

Так как поля $F^{i k}$ и $G^{i k}$ по отдельности удовлетворяют уравнениям Максвелла в пустоте, для тензоров $T_{F F}^{i k}, T_{G G}^{i k}$, а также $T^{i k}$ справедливы уравнения (4). Из формулы (8) следует, что уравнения (4) справедливы и для тензора $T_{F G}^{i k}$.

При вычислении потоков тензоров (8) через 3-поверхность $\Sigma$ мы будем исходить из предположения о квазиоднородности внешнего поля, т.е. будем считать величины $F^{i k}$ постоянными и вынесем их за знак интеграла. Ниже (см. п. 6.5) мы дадим количественную оценку допустимости такого приближения.

Примем естественное предположение об "упругом" характере взаимодействия частицы с полем: некоторое "собственное состояние" частицы не изменяется при движении, а энергия, возникаюшая при взаимодействии, связана исключительно с изменением скорости частицы. В системе сопутствующего наблюдателя построим вокруг частицы 2-сферу $S$ радиуса $\rho$. Предположим, что при некотором значении радиуса $\rho 4$-вектор энергии-импульса объема $V$, ограниченного $S$, имеет вид

$$
P^{i}=\{\mu, 0,0,0\}
$$

где $\mu$ - некоторая константа, не зависяшая от выбранной точки на мировой линии частищы. Тогда для энергии-импульса объема $V$ находим

$$
P^{i}=\mu u^{i}
$$

Более сложные гипотетические выражения для энергии-импульса частишы содержат не только скорость, но и ускорение и, возможно, более старшие производные [8].

Ниже мы увидим, что если для поля частицы справедливо выражение (7), то уравнение (10) имеет место лишь при единственном значении величины радиуса $\rho$, который будет выражен через заряд и массу частицы (см. (45)).

\section{4. ПОСТРОЕНИЕ ПОВЕРХНОСТИ ИНТЕГРИРОВАНИЯ}

Выберем некоторую точку $z^{i}$ на мировой линии частицы, перейдем в систему сопутствуюшего наблюдателя и построим 2-сферу $S$ радиуса $\rho$ с центром $z^{i}$. Припишем всем точкам $S$ временну́ю координату $x^{0}=z^{0}+\rho$. Таким образом, все точки $S$ оказываются на поверхности светового конуса с вершиной в выбранной точке $z^{i}$, ограничивающего область абсолютного будушего. 
2-поверхность $S$ является пересечением 3 -поверхности светового конуса и 3 -плоскости $x^{0}-z^{0}=\rho$. Легко видеть, что $S$ может быть определена системой инвариантных уравнений

$$
\begin{aligned}
\chi^{i} u_{i} & =\rho, \\
\chi^{i} \chi_{i} & =0,
\end{aligned}
$$

где $\chi^{i}=x^{i}-z^{i}-$ светоподобный вектор запаздывания светового сигнала из точки траектории $z^{i}, u^{i}-4$-скорость частишы в точке $z^{i}$.

Определим 3-поверхность $\Sigma$ как объединение 2-сфер $S$ фиксированного радиуса $\rho$, построенных для каждой точки мировой линии частицы.

Чтобы получить аналитическое выражение для 3-поверхности $\Sigma$, поступим следующим образом. Обозначим через $\tau$ собственное время частицы, являющееся естественной параметризацией мировой линии $z^{i}(\tau)$. Определим функцию 4-координат $\tau\left(x^{i}\right)$ как значение $\tau$ в точке пересечения поверхности светового конуса абсолютного прошлого точки $x^{i}$ с мировой линией частицы $z^{i}(\tau)$. Таким образом, $\tau\left(x^{i}\right)$ задается неявно уравнением

$$
\left(x^{i}-z^{i}(\tau)\right)\left(x_{i}-z_{i}(\tau)\right)=0 .
$$

Определим функцию $\chi^{i}\left(x^{k}\right)$ как вектор запаздывания сигнала из “точки излучения" $z^{i}(\tau)$ в “точку наблюдения" $x^{i}$ :

$$
\chi^{i}\left(x^{k}\right)=x^{i}-z^{i}\left(\tau\left(x^{k}\right)\right)
$$

Уравнение (11) и является искомым уравнением 3-поверхности $\Sigma$, если под $\chi^{i}$ понимать функцию координат, определенную во всем 4-пространстве, а под $u^{i}-4$-скорость частицы в точке $z^{i}(\tau)$. Из уравнений (13) и (14) видно, что $\chi^{i}$ - светоподобный вектор.

Производные функции $\tau\left(x^{i}\right)$ легко определить, дифференцируя уравнение (13):

$$
\partial_{k} \tau=\frac{\chi_{k}}{\chi^{i} u_{i}}
$$

Теперь вычисляем дифференциал уравнения (11):

$$
\left(u_{k}+\left(-1+\chi^{i} a_{i}\right) \partial_{k} \tau\right) d x^{k}=0
$$

где $a^{i}-4$-ускорение частицы в точке $z^{i}(\tau)$. Отсюда для 4-вектора нормали $k_{i} \mathrm{k} 3$-поверхности $\Sigma$ с учетом (11) получаем

$$
k_{i}=\frac{1}{\sqrt{1-2 \chi^{m} a_{m}}}\left(u_{i}+\left(-1+\chi^{l} a_{l}\right) \frac{\chi_{i}}{\rho}\right)
$$

Нормировочный множитель мы нашли из условия $k_{m} k^{m}=-1$. 


\section{5. ВЫЧИСЛЕНИЕ ИНТЕГРАЛА ПО ПОВЕРХНОСТИ ТРУБКИ}

Учитывая выражение (16), уравнение (3) можно переписать в виде

$$
P^{i}(\bar{S})-P^{i}(S)=-\int_{\Sigma} T^{i k}\left(u_{k}+\left(-1+\chi^{l} a_{l}\right) \frac{\chi_{k}}{\rho}\right) \frac{d V}{\sqrt{1-2 \chi^{m} a_{m}}}
$$

где $d V$ - элемент объема 3-поверхности $\Sigma$.

Рассмотрим $z^{i}$ и $\bar{z}^{i}$ - два близких события на мировой линии частицы, отделенные интервалом собственного времени $\delta \tau$ :

$$
\bar{z}^{i}=z^{i}+u^{i} \delta \tau
$$

Построим световые конусы абсолютного будушего с вершинами в 4-точках $z^{i}$ и $\bar{z}^{i}$ (см. рисунок). Применим уравнение (17) к 2-поверхностям $S$ и $\bar{S}$, возникающим при пересечении 3 -поверхности $\Sigma$ с 3 -поверхностями этих световых конусов, соответственно. Участок $\Sigma$, заключенный между сферами $S$ и $\bar{S}$, обозначим $\delta \Sigma$.

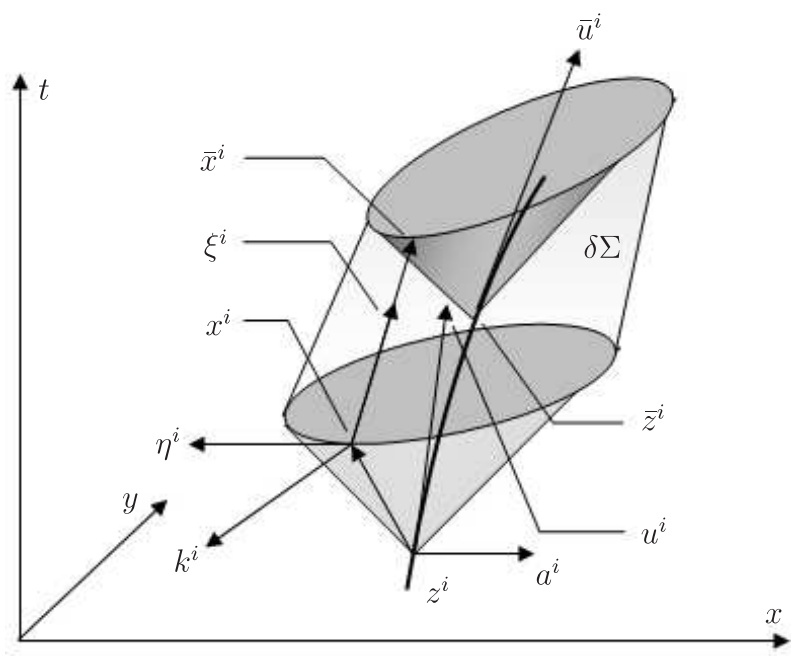

Выберем на 2-поверхности $S$ произвольную точку $x^{i}$ и построим три вектора $\xi^{i}, \eta^{i}$, $\zeta^{i}$, образующие ортогональный нормированный базис в 3 -пространстве, касательном к 3 -поверхности $\Sigma$ в точке $x^{i}$. Все три построенных вектора ортогональны вектору нормали $k^{i}$. Как видно из выражения (16), вектор $k^{i}$ лежит в 2-плоскости $U$, образуемой векторами $\chi^{i}$ и $u^{i}$. Мы выберем базисньй вектор $\xi^{i}$ также в 2-плоскости $U$, а векторы $\eta^{i}$ и $\zeta^{i}$ - ортогональными $U$. Легко убедиться, что вектор $\xi^{i}$ имеет вид

$$
\xi^{i}=\frac{1}{\sqrt{1-2 \chi^{m} a_{m}}}\left(u^{i}-\chi^{l} a_{l} \frac{\chi^{i}}{\rho}\right) .
$$

В качестве элемента интегрирования в уравнении (17) выберем параллелепипед с ребрами, лежашими на векторах $\xi^{i}, \eta^{i}, \zeta^{i}$. Найдем $l$ - длину ребра параллелепипеда, 
направленного вдоль $\xi^{i}$ и заключенного между 2-поверхностями $S$ и $\bar{S}$. Для этого найдем $\bar{x}^{i}$ - точку пересечения этого ребра и светового конуса точки $\bar{z}^{i}$ :

$$
\begin{gathered}
\bar{x}^{i}=x^{i}+l \xi^{i} \\
\left(\bar{x}^{i}-z^{i}-\bar{u}^{i} \delta \tau\right)\left(\bar{x}_{i}-z_{i}-\bar{u}_{i} \delta \tau\right)=0
\end{gathered}
$$

здесь $\bar{u}_{i}=u^{i}+\delta \tau \cdot a^{i}-4$-скорость частицы в момент $\bar{z}^{i}$. Оставляя члены первого порядка малости по $\delta \tau$ и учитывая уравнение (11), получим

$$
l=\delta \tau \sqrt{1-2 \chi^{m} a_{m}} .
$$

Теперь можно в уравнении (17) проинтегрировать вдоль направления $\xi^{i}$ и перейти к интегралу по 2-поверхности $S$ :

$$
\delta P^{i}=-\delta \tau \int_{S} T^{i l}\left(u_{l}+\left(-1+\chi^{m} a_{m}\right) \frac{\chi_{l}}{\rho}\right) d S .
$$

Далее преобразуем интеграл (23) к интегралу по пространственному телесному углу do. Это не тривиально, так как сфера $S$ не является обычной пространственной сферой, что хорошо видно из уравнения (11). Она сплюснута лоренцевым сокращением и не лежит в 3 -пространстве $t=$ const.

Поместим начало координат и начало отсчета времени в “точку излучения" $z^{i}$. Выберем полярную ось вдоль направления скорости частицы и введем полярную систему координат $t, R, \theta, \varphi$. Тогда для компонент $x^{i}$ мы получим (причем $\chi^{i}=x^{i}$ )

$$
x^{i}=(t, R \sin \theta \cos \varphi, R \sin \theta \sin \varphi, R \cos \theta),
$$

для компонент 4-скорости-

$$
u^{i}=\left(\frac{1}{\sqrt{1-v^{2}}}, 0,0, \frac{v}{\sqrt{1-v^{2}}}\right) .
$$

Уравнения 2-поверхности $S$ (11) и (12) принимают вид

$$
\begin{aligned}
t-R v \cos \theta & =\rho \sqrt{1-v^{2}}, \\
t & =R .
\end{aligned}
$$

Выберем базисные векторы $\eta^{i}, \zeta^{i}$ в виде

$$
\begin{aligned}
\eta^{i} & =\{-v \sin \theta,(\cos \theta-v) \cos \varphi,(\cos \theta-v) \sin \varphi,-\sin \theta\} \frac{1}{1-v \cos \theta}, \\
\zeta^{i} & =\{0,-\sin \varphi, \cos \varphi, 0\}
\end{aligned}
$$

Легко убедиться, что векторы $\xi^{i}, \eta^{i}$ и $\zeta^{i}$ образуют ортогональньй нормированный базис в 3-пространстве, проходящем через точку $x^{i}$ и ортогональном вектору $k^{i}$, определенному выражением (16). 
При малом изменении углов $\delta \theta$ и $\delta \varphi$ концы векторов $x^{i}$ испытывают малые изменения, скользя по поверхности сферы $S$. Дифференцируя (24) и (26), находим

$$
\delta x^{i}=\frac{\rho \sqrt{1-v^{2}}}{1-v \cos \theta} \eta^{i} \delta \theta+\frac{\rho \sqrt{1-v^{2}}}{1-v \cos \theta} \zeta^{i} \sin \theta \delta \varphi .
$$

Отсюда мы приходим к выражению для элемента интегрирования $d S$ в интеграле $(23)$ через дифференциалы углов $d \theta$ и $d \varphi$ :

$$
d S=\frac{\rho^{2}\left(1-v^{2}\right)}{(1-v \cos \theta)^{2}} \sin \theta d \theta d \varphi .
$$

Вычислим некоторые интегралы по поверхности $S$, которые пригодятся в дальнейшем:

$$
\begin{aligned}
\oint_{S} d S & =\int_{0}^{2 \pi} d \varphi \int_{0}^{\pi} \frac{\rho^{2}\left(1-v^{2}\right)}{(1-v \cos \theta)^{2}} \sin \theta d \theta=4 \pi \rho^{2}, \\
\oint_{S} \chi^{i} d S & =4 \pi \rho^{3} u^{i}, \\
\oint_{S} \chi^{i} \chi^{k} d S & =\frac{4 \pi \rho^{4}}{3}\left(4 u^{i} u^{k}-g^{i k}\right), \\
\oint_{S} \chi^{i} \chi^{k} \chi^{l} d S & =8 \pi \rho^{5} u^{i} u^{k} u^{l}-\frac{4 \pi \rho^{5}}{3}\left(g^{i k} u^{l}+g^{i l} u^{k}+g^{k l} u^{i}\right) .
\end{aligned}
$$

Теперь преобразуем тензор энергии-импульса (5). Для этого необходимо подставить в выражение (5) сумму $F^{i k}+G^{i k}$, раскрыть скобки, разбить результат на три слагаемых в соответствии с (8), подставить вместо $G^{i k}$ его выражение (7) (которое на поверхности $\Sigma$ ввиду уравнения (11) имеет достаточно простой вид), затем свернуть полученные выражения с выражением в круглых скобках под интегралом в формуле (23), которое мы обозначим через $\psi_{l}$ :

$$
\psi_{l}=u_{l}+\left(-1+\chi^{m} a_{m}\right) \frac{\chi_{l}}{\rho} .
$$

Опуская эти элементарные, хотя и несколько громоздкие преобразования, выпишем сразу окончательные результаты ${ }^{1)}$ :

$$
\begin{aligned}
T_{F F}^{i l} \psi_{l}= & \frac{1}{4 \pi}\left(-F^{i m} F_{m}^{l}+\frac{1}{4} g^{i l} F_{k m} F^{k m}\right)\left(u_{l}+\left(-1+\chi^{m} a_{m}\right) \frac{\chi_{l}}{\rho}\right) \\
T_{F G}^{i l} \psi_{l}= & -\frac{1}{4 \pi} F_{l}^{i}\left(\frac{e}{\rho^{2}} u^{l}\left(1-\chi^{m} a_{m}\right)+\frac{e}{\rho} a^{l}\right)+ \\
& +\frac{1}{4 \pi} \frac{e}{\rho^{2}} F_{k l}\left(\chi^{k} u^{i} a^{l}+\chi^{i} u^{l} a^{k}+\chi^{l} u^{k} a^{i}\right) \\
T_{G G}^{i l} \psi_{l}= & \frac{e^{2}}{8 \pi \rho^{4}} u^{i}\left(1-2 \chi^{m} a_{m}\right)+\frac{e^{2}}{4 \pi \rho^{3}}\left(a^{i}-\chi^{i} a^{k} a_{k}\right)-
\end{aligned}
$$

\footnotetext{
1) Изящный способ вычисления выражений вида (35)-(37) предложен в книге [17], §15.
} 


$$
-\frac{e^{2}}{8 \pi \rho^{5}}\left(\chi^{i}-3 \chi^{i} \chi^{n} a_{n}+2 \chi^{i} \chi^{m} \chi^{n} a_{m} a_{n}\right)
$$

Вычисляем интегралы выражений (35)-(37) по поверхности $S$ с помощью формул (30)-(33), затем суммируем и окончательно находим

$$
\delta P^{i}=\delta \tau\left(-\frac{\rho^{3}}{3} F^{i l} F_{k l} a^{k}+\frac{\rho^{3}}{12} F_{l m} F^{l m} a^{i}+e F^{i l} u_{l}+e \rho F^{i l} a_{l}-\frac{e^{2}}{2 \rho} a^{i}+\frac{2 e^{2}}{3} u^{i} a^{k} a_{k}\right) .
$$

\section{6. ВЫВОД УРАВНЕНИЯ ДВИЖЕНИЯ ЧАСТИЦ}

6.1. В выражении (38) под $F^{i k}$ мы условимся понимать значение напряженностей внешнего электромагнитного поля в точке, где находится частица. Интеграл (17) вычисляется по поверхности $\Sigma$, где поля $F^{i k}$ и $G^{i k}$ полностью определены. Чтобы в дальнейшем не возникали парадоксы, мы не рассматриваем значений реальных напряженностей $F^{i k}+G^{i k}$ в точке, где находится частица.

Учитывая предположение о квазиоднородности поля $F^{i k}$ в окрестности частицы, можно математически непрерывно продолжить функции $F^{i k}$ в точку, где находится частица, и далее использовать это значение.

6.2. Пренебрежем первыми двумя слагаемыми в выражении (38), квадратичными по компонентам внешнего поля $F^{i k}$, которые будем считать достаточно малыми. Ниже (в п. 6.5) мы получим количественную оценку допустимости такого приближения. Пятое слагаемое представляет собой “электромагнитную массу” частищы. Мы перенесем его в левую часть. Используя уравнение (10), выразим $\delta P^{i}$ через 4-ускорение частицы: $\delta P^{i}=\mu a^{i} \delta \tau$, и приведем выражение (38) к виду

$$
m a^{i}=e F^{i l} u_{l}+e \rho F^{i l} a_{l}+\frac{2 e^{2}}{3} u^{i} a^{k} a_{k},
$$

где через $m$ обозначена величина, которую мы должны отождествить с наблюдаемой массой частишы ${ }^{2}$ :

$$
m=\mu+\frac{e^{2}}{2 \rho}
$$

Свернув обе части формулы (39) с $a_{i}$, найдем выражение для инвариантного квадрата 4-ускорения

$$
a^{i} a_{i}=\frac{e}{m} F^{i l} u_{l} a_{i}
$$

и подставим выражение (41) в (39):

$$
m a^{i}=e F^{i l} u_{l}+e \rho F^{i l} a_{l}+\frac{2 e^{3}}{3 m} u^{i} F^{k l} a_{k} u_{l} .
$$

\footnotetext{
${ }^{2)}$ В работе [7], опубликованной впервые в 1923 г., для “электромагнитной массы” частицы с зарядом, распределенным равномерно по сфере радиуса $\rho$, получено выражение $m=(1 / 2) e^{2} /\left(\rho c^{2}\right)$ из вариационного принципа. В работе [8] (1938г.) выражение (40) получено способом, аналогичным использованному нами. Несмотря на это, в литературе приходится встречаться со старым выражением для “электромагнитной массы” $m=(2 / 3) e^{2} /\left(\rho c^{2}\right)$, полученным в рамках нерелятивистской теории [6] и противоречащим релятивистскому соотношению массы и энергии $\mathcal{E}=m c^{2}$.
} 
Свернув последнее уравнение с $u_{i}$, найдем, что оно разрешимо при условии

$$
m=\frac{2 e^{2}}{3 \rho} .
$$

Из уравнений (40) и (43) найдем выражения для затравочной массы $\mu$ и радиуса трубки $\rho$ :

$$
\begin{aligned}
& \mu=\frac{m}{4}, \\
& \rho=\frac{2 e^{2}}{3 m} .
\end{aligned}
$$

Подставив выражение (45) в формулу (39), получим

$$
m a^{i}=e F^{i l} u_{l}+\frac{2 e^{3}}{3 m} F^{i l} a_{l}+\frac{2 e^{2}}{3} a^{k} a_{k} u^{i} .
$$

Уравнение (46) отличается от уравнения ЛД (1) всего одним членом, но это отличие принципиально, так как (46) не содержит вторых производных скорости.

6.3. Выражения (44) и (45) требуют физического осмысления. Пока мы не пришли к (45), наши рассуждения были справедливы для каждого значения величины $\rho$. Однако выяснилось, что уравнение (42), которое по физическому смыслу есть уравнение баланса энергии на поверхности трубки $\Sigma$, разрешимо только при одном-единственном значении $\rho$. Этого достаточно для решения стояшей перед нами сейчас задачи - доказать, что уравнение (46) находится в строгом соответствии с законом сохранения энергии.

В самом деле, условие баланса энергии-импульса выполнено на любой 3-поверхности $\Sigma^{\prime}$, если оно выполнено хотя бы на одной поверхности $\Sigma$, окружаюшей частицу, в силу равенства нулю 4-дивергенции тензора энергии-импульса поля в свободном от частиц объеме.

Таким образом, для вычисления энергии-импульса внутри произвольной поверхности, заключаюшей частицу, следует в системе сопутствуюшего наблюдателя окружить частицу 2-сферой $S$ радиуса $\rho$ из выражения (45) и приписать энергии объема $V$, ограниченного $S$, величину $m c^{2} / 4$, а затем вычислить энергию искомого объема, добавляя (или вычитая, если рассматриваемая поверхность лежит внутри $S$ ) значение энергии-импульса, которое в сопутствуюшей системе может быть вычислено как

$$
P^{i}=\int_{V^{\prime}} T^{i 0} d V
$$

где интеграл берется по объему $V^{\prime}$ с вырезанной сферой радиуса $\rho$.

Здесь интересно было бы рассмотреть следуюший вопрос: какой вид приобретает уравнение баланса энергии-импульса для поверхности $\Sigma$, построенной для значений радиуса $\rho$, отличных от (45)? Вопрос этот, выходяший за рамки данной статьи, заслуживает отдельного подробного исследования. 
6.4. Ведем обозначение

$$
\beta=\frac{2 e^{3}}{3 m^{2}}
$$

и перепишем выражение (42) в виде системы линейных уравнений относительно $a_{i}$ :

$$
\left(g^{i l}-\beta F^{i l}-\beta u^{i} F^{l k} u_{k}\right) a_{l}=\frac{e}{m} F^{i l} u_{l} .
$$

Матрицу, действующую на вектор $a_{l}$ в левой части $(49)$, обозначим через $D^{i l}$ :

$$
D^{i l}=g^{i l}-\beta F^{i l}-\beta u^{i} F^{l k} u_{k}
$$

Так как $D^{i l}$ является 4-тензором относительно преобразований Лоренца, то определитель ее - псевдоскаляр. Если определитель отличен от нуля в одной системе отсчета, то он отличен от нуля и в любой другой. Вычислим этот определитель в сопутствуюшей системе:

$$
\left|D^{i l}\right|=\left|\begin{array}{cccc}
1 & 0 & 0 & 0 \\
\beta E_{x} & -1 & -\beta H_{z} & \beta H_{y} \\
\beta E_{y} & \beta H_{z} & -1 & -\beta H_{x} \\
\beta E_{z} & -\beta H_{y} & \beta H_{x} & -1
\end{array}\right|=-1-\beta^{2} H^{2} .
$$

Отсюда видно, что определитель системы (49) заведомо отличен от нуля во всех системах отсчета, следовательно, система (49) имеет единственное решение. Представим его в виде

$$
m a_{i}=e F_{i m} u^{m}+e \beta \frac{\left(-F^{k l} F_{k i}+F^{m n} F_{m j} u^{j} u_{n} \delta_{i}^{l}\right)\left(u_{l}+\beta F_{l m} u^{m}\right)}{1+\beta^{2}\left(F_{m n} F^{m n} / 2-F^{m n} F_{m j} u^{j} u_{n}\right)} .
$$

Для вывода выражения (52) мы воспользовались формулой Крамерса, представив решение системы (49) как отношение двух определителей, а затем вычислили эти определители с помощью техники тензорной алгебры. Мы не станем приводить здесь эти громоздкие и по сушеству элементарные вычисления, а убедимся в справедливости выражения (52) следуюшим образом. Поскольку (52) есть равенство двух 4-векторов, то достаточно проверить его в одной системе отсчета.

Проверим его в сопутствуюшей системе, где матрица $D^{i l}$ имеет вид (51). Несложно убедиться, что выражение (52) удовлетворяет тождеству

$$
a_{i} u^{i} \equiv 0
$$

Для этого достаточно свернуть (52) с $u^{i}$ и учесть, что вследствие антисимметрии тензора $F^{i k}$ имеет место тождество

$$
F_{l m} u^{m} F^{k l} F_{k i} u^{i}=0 .
$$

Из тождества (53) сразу следует, что в сопутствующей системе

$$
a_{0}=0
$$


Рассмотрим теперь пространственные компоненты (52) в сопутствующей системе. Нам будет удобно использовать следующее свойство: пространственные компоненты свертки тензора $F_{l}^{i}$ с произвольным контравариантным вектором $b^{l}$ образуют вектор $N^{i}=F_{l}^{i} b^{l}$, пространственные компоненты которого определяются формулой

$$
\vec{N}=b^{0} \vec{E}+\vec{b} \times \vec{H}
$$

Перейдем к трехмерным обозначениям, предварительно подняв индекс $i$ в выражении (52):

$$
m \vec{a}=e \vec{E}+e \beta \frac{\vec{E} \times \vec{H}+\beta\left(L^{0} \vec{E}+\vec{L} \times \vec{H}-E^{2} \vec{E}\right)}{1+\beta^{2} H^{2}}
$$

где мы обозначили $L^{k}=F_{l}^{k} F_{m}^{l} u^{m}$, а кроме того, использовали соотношения

$$
F^{m n} F_{m n}=-2 E^{2}+2 H^{2}, \quad F^{m n} F_{m j} u^{j} u_{n}=-E^{2} .
$$

Из формулы (56) видно, что

$$
L^{0}=E^{2}, \quad \vec{L}=\vec{E} \times \vec{H} .
$$

Подставляя уравнения (59) в выражение (57) и упрощая, получаем выражение для ускорения частицы ${ }^{3)}$ в системе сопутствующего наблюдателя:

$$
m \vec{a}=e \frac{\vec{E}+\beta \vec{E} \times \vec{H}+\beta^{2} \vec{H}(\vec{E} \cdot \vec{H})}{1+\beta^{2} H^{2}} .
$$

Пространственные компоненты уравнения (49) в сопутствуюшей системе отсчета в обозначениях векторного анализа имеют вид

$$
\vec{a}-\beta\left(a^{0} \vec{E}+\vec{a} \times \vec{H}\right)=\frac{e}{m} \vec{E}
$$

Несложно убедиться, что 4-вектор $a^{i}$, определенный соотношениями (55) и (60), , является решением системы уравнений (61). Таким образом, мы доказали, что выражение (52) определяет единственное решение системы уравнений (49) в системе отсчета сопутствуюшего и, следовательно, произвольного наблюдателя.

\footnotetext{
3) Через $\vec{a}$ мы обозначили 3-вектор, составленный из пространственных компонент 4-вектора ускорения $a^{i} \equiv d u^{i} / d \tau$. Вектор $\vec{a}$ в произвольной системе координат не совпадает с $d \vec{v} / d t-3$-вектором ускорения нерелятивистской механики. Однако в сопутствующей системе отсчета, как легко показать, эти величины равны; следовательно, мы можем понимать (60) как выражение для силы, действующей на покоящуюся частицу со стороны электромагнитного поля.
} 
6.5. Уравнение (52) можно рассматривать как уравнение движения заряженной частищы. Первое слагаемое в правой части есть сила Лоренца, а второе - дополнительная релятивистская поправка. Мы остережемся до специального исследования называть второй член “силой радиационного трения" или "реакцией излучения", так как не вся работа поля, соответствующая второму слагаемому в (52), идет на излучение электромагнитных волн, необратимо уносяших энергию на бесконечность. Чтобы вычленить из уравнения (52) консервативную составляющую и силу радиационного трения, необходимо вычислить поток энергии, уносимой излучением на бесконечность, что в общем случае не очевидно.

Для известных элементарных частиц величина $\beta$ крайне мала. В частности, для электрона, переходя к гауссовым единицам, находим ( $c$ - скорость света)

$$
\beta_{\mathrm{e}}=\frac{2 e^{3}}{3 m^{2} c^{4}}=1.1 \cdot 10^{-16}{ }^{-1} .
$$

Для выяснения физического смысла величины $\beta$ введем величину $E_{\mathrm{cl}}$, равную теоретическому значению напряженности электрического поля на расстоянии от частицы, равном классическому радиусу электрона $r_{\mathrm{cl}}$ :

$$
E_{\mathrm{cl}}=\frac{e}{r_{\mathrm{cl}}^{2}}=\frac{m^{2} c^{4}}{e^{3}}=6 \cdot 10^{15} \text { Э. }
$$

Из формул (62) и (63) видно, что

$$
E_{\mathrm{cl}}=\frac{2}{3} \beta^{-1}
$$

Даже вблизи поверхности нейтронных звезд обычно предполагают поля на два-три порядка меньше, чем $E_{\mathrm{cl}}[1]$. Кроме того, образованию таких сильных полей препятствуют квантовые явления, в частности, образование электрон-позитронных пар, которые начинают играть определяющую роль при условии разности потенциалов, превосходящей энергию покоя частищ, на комптоновской длине. Обозначив через $E_{\text {qu }}$ критическую для этого процесса напряженность электрического поля, находим

$$
e E_{\mathrm{qu}} \frac{\hbar}{m c} \sim 2 m c^{2}
$$

Из формул (63) и (65) получаем

$$
E_{\mathrm{qu}} \sim \frac{2 m^{2} c^{3}}{e \hbar}=2 E_{\mathrm{cl}} \frac{e^{2}}{\hbar c}=\frac{2}{137} E_{\mathrm{cl}}
$$

т.е. при полях, на два порядка меньших, чем $E_{\mathrm{cl}}$, определяющую роль начинают играть квантовые явления.

Таким образом, во всех практических случаях, когда квантовые эффекты малы и применима классическая электродинамика, напряженности полей не превышают $E_{\text {qu }}$ и, значит, всегда можно считать $E \ll E_{\text {сl }}$; следовательно,

$$
\beta F^{i k} \ll 1
$$


Вернемся теперь к уравнению (38), где мы пренебрегли первыми двумя слагаемыми в правой части по сравнению с левой частью, т.е. исходили из предположения, что

$$
\frac{\rho^{3}}{m} F^{i k} F^{l m} \ll 1
$$

Как легко видеть из выражений (45) и (48), левая часть неравенства (68) имеет порядок $\left(\beta F^{i k}\right)^{2}$ и мала в силу условия (67). Причем, пренебрегая членами второго порядка малости, мы сохранили четвертое слагаемое в уравнении $(38)$, которое является поправкой первого порядка малости по $\beta F^{i k}$. Обратим внимание, что в данном случае мы не ограничиваем лоренц-фактор частицы $u^{0}$, в связи с чем величину $\beta F^{i k} u^{l}$ не считаем малой.

Нам осталось исключить из выражения (52) (которое есть точное решение уравнения (49)) члены второго порядка малости по $\beta F^{i k}$ :

$$
m a^{i}=e \frac{F^{i m} u_{m}+\beta F^{i k} F_{k l} u^{l}+\beta u^{i} F^{m n} F_{m j} u^{j} u_{n}}{1-\beta^{2} F^{m n} F_{m j} u^{j} u_{n}} .
$$

Уравнение (69) и есть искомое уравнение движения классической заряженной частицы. Оно справедливо при выполнении условия (67). В противном случае (гипотетические заряженные частицы с массой много меньше массы электрона или сверхсильные поля на ранних стадиях Большого взрыва) теряют силу уравнения (69) и (52) и необходимо учитывать вклад двух первых слагаемых в (38).

Уравнение (38) получено в предположении квазиоднородности внешнего поля $F^{i k}$. Для количественной оценки влияния неоднородности поля $F^{i k}$ на движение частицы рассмотрим движение в поле волны с волновым вектором $k_{i}$. Тогда величины $F^{i k}$ в уравнении (36) следует считать пропорциональными $e^{i k_{i} \chi^{i}}$, что после интегрирования по координатам приведет к появлению в уравнении (38) дополнительных членов порядка $\rho k_{i} u^{i}$. Отсюда находим, что как (38), так и следующие из него уравнения (46) и (69) имеют силу при условии

$$
\lambda \gg \frac{e^{2}}{m c^{2}} u^{0},
$$

где $\lambda$ - длина падающей электромагнитной волны. Таким образом, в задачах о взаимодействии ультрарелятивистской частицы с внешним высокочастотным полем необходимо учитывать соответствующие поправки к уравнению (38).

\section{7. ОБСУЖДЕНИЕ РЕЗУЛЬТАТОВ}

Рассмотрим частные случаи движения частицы во внешних полях.

7.1. Пусть частица покоится $-v=0$. Исключая из выражения (60) члены второго порядка по $\beta H$, получаем

$$
\frac{d \vec{p}}{d t}=e \vec{E}+e \beta[\vec{E} \times \vec{H}]
$$

Так как второй член в правой части уравнения (70) мал по сравнению с первым, то он может представлять интерес только при усреднении по времени, если среднее значение 
$\vec{E}$ исчезает. При рассеянии электромагнитной волны свободными частицами (томсоновское рассеяние) в гауссовых единицах находим

$$
\frac{\overline{d \vec{p}}}{d t}=\frac{2}{3}\left(\frac{e^{2}}{m c^{2}}\right)^{2} \overline{[\vec{E} \times \vec{H}]} .
$$

Выражение (71) получено в книге [9] (§ 78) из соображений баланса импульса: падаюшая волна теряет энергию и связанный с энергией импульс, излучаемая же частицей волна уносит энергию, но не уносит импульса. Отсюда делается вывод, что теряемая часть импульса падающей волны передается частище. Количественный расчет теряемого в единицу времени импульса приводит к выражению (71).

Любопытно отметить, что, согласно уравнению (70), дополнительная релятивистская сила действует на покояшуюся частицу.

7.2. Рассмотрим движение субрелятивистской частицы в магнитном поле. Упростим уравнение (69), предполагая $\vec{E}=0$ и $\beta H\left(u^{0}\right)^{2} \ll 1$ :

$$
\frac{d \vec{p}}{d t}=e[\vec{v} \times \vec{H}]+e \beta[[\vec{v} \times \vec{H}] \times \vec{H}]-e \beta \frac{\vec{v}[\vec{v} \times \vec{H}]^{2}}{1-v^{2}} .
$$

Умножим обе части уравнения (72) скалярно на $\vec{v}$ :

$$
\frac{d K}{d t}=e \beta \frac{(\vec{v} \cdot \vec{H})^{2}-v^{2} H^{2}}{1-v^{2}}=-e \beta \frac{v_{\perp}^{2} H^{2}}{1-v^{2}} ;
$$

здесь $K=m / \sqrt{1-v^{2}}$ - релятивистская кинетическая энергия частицы.

Мы видим из выражения (73), что при наличии $v_{\perp}-$ перпендикулярной магнитному полю составляюшей скорости частицы - частица медленно теряет кинетическую энергию, причем величина ее потерь в единицу времени равна интенсивности магнитотормозного излучения (см. [9], § 74).

7.3. Рассмотрим релятивистскую частицу такую, что $u^{0} \gg 1$, но $\beta F^{i k}\left(u^{0}\right)^{2} \ll 1$.

Опустим в числителе уравнения (69) второй член, мальй по сравнению с третьим в силу $u^{0} \gg 1$; затем, пренебрегая членами второго порядка по $\beta F^{i k}$, приведем $(69) \mathrm{k}$ виду

$$
m a^{i}=e F^{i m} u_{m}+\frac{2 e^{2}}{3} u^{i} a^{k} a_{k} .
$$

Дополнительное слагаемое в формуле (74) совпадает (с обратным знаком, естественно) с известным выражением для мошности $I$ дипольного излучения (см. [9], $\S \S 67,73)$. Таким образом, мы можем интерпретировать уравнение (74) как торможение излучением в ультрарелятивистском случае. Пространственные компоненты (74) дают

$$
\frac{d \vec{p}}{d t}=q \vec{E}+q[\vec{v} \times \vec{H}]-\vec{v} I .
$$

Физически уравнение (75) выражает тот факт, что в ультрарелятивистском случае все излучение частицы направлено вперед, вдоль ее скорости, а отдача равна полному импульсу излучения, взятому с обратным знаком. 
7.4. Рассмотрим следующий диапазон энергий:

$$
\beta F^{i k}\left(u^{0}\right)^{2} \gg 1, \quad \text { но } \quad \beta F^{i k} u^{0} \ll 1 .
$$

Определяющим движение здесь становится третье слагаемое в числителе уравнения (69). Пренебрегая первым и вторым слагаемыми, а также считая знаменатель близким к единице, получим

$$
m a^{i}=e \beta F^{m n} F_{m l} u^{l} u_{n} u^{i} .
$$

Характер движения в этих условиях исследован Померанчуком [18] (см. также [9], $\S 76$; [1], гл.4). При делении на $u^{0}$ временно́й компоненты уравнений (77) получается, что потери частищей энергии на единице пути пропорциональны квадрату энергии частицы, откуда делается вывод, что после пролета частицы через данное поле ее кинетическая энергия не может превьшшать некоторого критического значения $K_{\max }$.

7.5. Диапазон сверхбольших энергий: $\beta F^{i k} u^{0} \gg 1$.

Исключим и из числителя, и из знаменателя уравнения (69) члены, не содержашие $u^{i}$ :

$$
m a^{i}=-\frac{e}{\beta} u^{i} .
$$

На частицу действует сила торможения, не зависящая от величины и направления электромагнитного поля; присутствие поля играет как бы роль катализатора. Потеря кинетической энергии частишы описывается законом ( $c$ - скорость света)

$$
K=K_{\mathrm{st}} \exp \left(-\frac{3 m c^{3}}{2 e^{2}} \tau\right)
$$

где $\tau$ - интервал собственного времени частищы.

Любопытно сравнить выражение (79) с известным "самоускоряюшимся решением" [8](см. также [9], § 75; [1], гл. 2), содержашим точно тот же показатель экспоненты, но с обратным знаком.

Однако физический смысл соотношений (78), (79) совершенно иной: выражение (78) устанавливает принципиальный предел количества энергии, теряемой частицей за единицу времени (или на единице пути), в каком бы сильном поле частица ни двигалась.

С помошью формулы (64) уравнение (78) перепишем в виде

$$
m a^{i}=-\frac{3}{2} e E_{\mathrm{cl}} u^{i} .
$$

Рассматривая уравнение (80), видим, что через величину $E_{\mathrm{cl}}$ выражается максимальное теоретически возможное "действующее" на частицу поле, вызывающее движение с максимально возможньм для данного типа частиц ускорением и максимально возможным темпом потери энергии.

Разделив на $u^{0}$ временну́ю компоненту уравнения (78), найдем предельное значение интенсивности излучения сверхэнергичной частищы:

$$
I_{\lim }=\frac{3 m^{2} c^{5}}{2 e^{2}}=1.31 \cdot 10^{17} \frac{\text { эрг }}{\mathrm{c}},
$$

5 Теоретическая и математическая физика, т. 143, № 1, 2005 г. 
где численное значение получено для заряда и массы электрона.

Проходя расстояние порядка комптоновской длины, такая частица теряет энергию

$$
\Delta K_{e} \sim I_{\lim } \frac{\hbar}{m c^{2}} \sim m c^{2} \frac{\hbar c}{e^{2}} \sim 137 m c^{2}
$$

т.е. такие гипотетические частицы теряют энергию в форме множественного рождения электрон-позитронных пар. Мы видим, что при обсуждении возможности сушествования в природе частиц сверхвысоких энергий необходимо учитывать квантовые эффекты.

Для данной нашей работы важно отметить, что с внутренними противоречиями в классической электродинамике мы не сталкиваемся и в диапазоне сверхвысоких энергий.

Благодарности. Автор благодарен С. С. Герштейну как за содержательные дискусси, так и за конкретную помошь при написании данной статьи. Автор признателен Б. М. Болотовскому и Л. А. Максимову за внимательное отношение к работе.

\section{Список литературы}

[1] В. Л. Гинзбург. Теоретическая физика и астрофизика. М.: Наука, 1987.

[2] Дж. Джексон. Классическая электродинамика. М.: Мир, 1965.

[3] Н. П. Клепиков. УФН. 1985. Т. 146. Вып.2. С. 317.

[4] J. L. Jimenez, I. Campos. Found. Phys. Lett. 1999. V. 12. № 2. P. 127.

[5] H. A. Lorentz. Arch. Neer. Sci. Exact. Nat. 1892. V. 25. P. 363.

[6] M. Abraham. Ann. Phys. 1903. V. 10. P. 105.

[7] Э. Ферми. Собрание научных трудов. Т. 1. М.: Наука, 1982. С. 73.

[8] P. A. M. Dirac. Proc. Roy. Soc. A. 1938. V. 167. P. 148.

[9] Л. Д. Ландау, Е. М. Лифииц. Теория поля. М.: Наука, 1973.

[10] J. A. Heras. Phys. Lett. A. 2003. V. 314. P. 272; M. Ribaric, L. Sustersic. Phys. Lett. A. 2002. V. 295. P. 318; S. D. Bosanac. J. Phys. A. 2001. V. 34. P. 473; H. D. Zee. Found. Phys. Lett. 1999. V. 12. № 2. P. 193; E. Comay. J. Phys. A. 1996. V. 29. P. 2111; C. Teitelboim, D. Villarroel, Ch. G. van Weert. Riv. Nouvo Cimento. 1980. V. 3. P. 9.

[11] Б. П. Косяков. ТМФ. 1999. Т. 119. С. 119; D. V. Gal'tsov. Phys. Rev. D. 2002. V. 66. P. 025016; P. O. Kazinski, S. L. Lyakhovich, A. A. Sharapov. Phys. Rev. D. 2002. V. 66. P. 025017.

[12] J. A. E. Roa-Neri, J. L. Jimenez. Found. Phys. 2002. V. 32. № 10. P. 1617; F. Rohrlich. Phys. Lett. A. 2002. V. 295. P. 320.

[13] F. Rohrlich. Phys. Lett. A. 2001. V. 283. P. 276.

[14] H. Spohn. Europhys. Lett. 2000. V. 50. P. 287; A. Bernui. Found. Phys. 2000. V. 30. P. 121.

[15] P. O. Kazinski, A. A. Sharapov. Class. Q. Grav. 2003. V. 20. P. 2715.

[16] A. Эйнитейн. Собрание научных трудов. Т. 2. М.: Наука, 1966. С. 674.

[17] Ю. В. Новожилов, Ю. А. Яппа. Электродинамика. М.: Наука, 1978.

[18] И. Я. Померанчук. ЖЭЭТ. 1939. Т. 9. С. 915. 